\title{
Prevalência de casos de leptospirose no Estado do Pará no período de 2007 a 2019
}

\author{
Prevalence of leptospirosis cases without State of Pará without period from 2007 to 2019 \\ Prevalencia de casos de leptospirosis en el Estado de Pará en el periodo de 2007 a 2019
}

Recebido: 22/09/2021 | Revisado: 28/09/2021 | Aceito: 30/09/2021 | Publicado: 02/10/2021

\author{
Danielle da Silva Cruz \\ ORCID: https://orcid.org/0000-0003-3503-6858 \\ Centro Universitário da Amazônia, Brasil \\ E-mail: danielle.silva.cruz001 @ gmail.com \\ Randria Leliane Cerdeira Moreira \\ ORCID: https://orcid.org/0000-0002-5998-2147 \\ Centro Universitário da Amazônia, Brasil \\ E-mail: randrialeliane10@gmail.com \\ Ingrid da Silva Leite \\ ORCID: https://orcid.org/0000-0002-2627-2675 \\ Centro Universitário da Amazônia, Brasil \\ E-mail: ingridleitestm@gmail.com
}

\begin{abstract}
Resumo
O objetivo neste trabalho foi investigar a prevalência de casos de leptospirose no estado do Pará no período de 2007 a 2019. Realizou-se um estudo descritivo e exploratório, por meio de uma retrospectiva epidemiológica da prevalência da Leptospirose no estado do Paraense. Os dados relacionados aos casos de leptospirose foram obtidos por meio do portal do Sistema de Informação de Agravos de Notificação online (SINAN Online), no período de 2007 a 2019, no Estado do Pará. Realizou-se a análise dos casos por meio do número de casos notificados, sendo considerado as variáveis: sexo, faixa etária, escolaridade e o critérios de confirmação. Os dados foram tabulados e organizados em planilhas, sendo expostos os dados percentuais referentes a cada análise. Neste estudo, foi possível identificar que o ano de maior ocorrência de casos foi 2014 com elevado número de casos confirmados. O grupo etário mais evidente foi pessoas do sexo masculino, com faixa etária de 20 a 39 anos, sendo o critério de confirmação clinico-laboratorial o mais adotado.
\end{abstract}

Palavras-chave: Critério de confirmação; Sexo; Faixa etária.

\begin{abstract}
The aim of this work was to investigate the epidemiological aspects; gender, age group and criteria for confirming leptospirosis in humans in the state of Pará, from 2007 to 2019. A descriptive and exploratory study was carried out through an epidemiological retrospective of the prevalence of leptospirosis in the state of Pará. Data related to leptospirosis cases were obtained through the portal of the Information System for Notifiable Diseases online (SINAN Online), in the period from 2007 to 2019, in the State of Pará. The analysis of the cases was carried out using the number of notified cases, considering the variables: gender, age, education and confirmation criteria. Data were tabulated and organized in spreadsheets, with percentage data for each analysis being displayed. In this study, it was possible to identify that the year with the highest occurrence of cases was 2014 with a high number of confirmed cases. The most evident age group was males, aged between 20 and 39 years, with the criterion for clinical and laboratory confirmation being the most adopted.
\end{abstract}

Keywords: Confirmation criteria; Gender; Age group.

\section{Resumen}

El objetivo de este estudio fue investigar la prevalencia de casos de leptospirosis en el estado de Pará de 2007 a 2019. Se realizó un estudio descriptivo y exploratorio mediante una retrospectiva epidemiológica de la prevalencia de leptospirosis en el estado de Pará. Los datos relacionados con los casos de leptospirosis se obtuvieron a través del portal del Sistema de Información de Enfermedades de Notificación en Línea (SINAN Online), en el período de 2007 a 2019, en el Estado de Pará. De casos notificados, considerando las variables: sexo, edad, educación y criterios de confirmación. Los datos se tabularon y organizaron en hojas de cálculo, mostrando los datos porcentuales de cada análisis. En este estudio se pudo identificar que el año con mayor ocurrencia de casos fue 2014 con un alto número de casos confirmados. El grupo de edad más evidente fue el del sexo masculino, con edades comprendidas entre los 20 y los 39 años, siendo el criterio de confirmación clínica y de laboratorio el más adoptado.

Palabras clave: Criterios de confirmación; Sexo; Grupo de edad. 


\section{Introdução}

A leptospirose é uma doença zoonótica ocasionada pela bactéria Leptospira, de ocorrência mundial, acomete animais silvestres e domésticos e o homem, sendo relatada a maior incidência de casos em pacientes com idade superior a 15 anos de ambos os sexos (Kawaguchi et al., 2008). Em diversas regiões comporta-se em caráter sazonal e epidêmico, favorecidos em países com clima tropical e com alta índice pluvial em períodos do ano (Picardeau, 2017; Soo et al., 2020).

Dessa maneira, o Brasil tornou-se um dos países mais endêmicos para a leptospirose, impulsionado pelas condições climáticas, socioeconômicas e pela falta de saneamento básico, sendo as regiões Sul, Sudeste e Nordeste as que apresentam o maior índice de casos registrados, em contrapartida as regiões com menores índices foram, Centro-Oeste e Norte, sendo o estado do Pará o que apresentou menor números de casos registrados, entretanto, a sintomatologia pode confundir com outras enfermidades, como a dengue, gripe e hepatite viral, pois ambas apresentam febre, dor abdominal e cefaleia, resultando na subnotificação da leptospirose (Rodrigues et al., 2005; Lupi et al., 2007).

No ano de 2015, por meio do Sistema de Informação de Agravos de Notificação SINAN/SVS/MS de casos e óbitos confirmados da leptospirose, notou-se que a região Sul apresentou 1192 casos e 79 óbitos, seguido pela região, com Sudeste 699 casos e 88 óbitos, Nordeste 338 casos e 50 óbitos, Centro-Oeste 54 casos e 7 óbitos, na região Norte 1188 casos, colocando o Pará com 103 casos e 20 óbitos, sendo os casos relatados em ambos os sexos, com maior predominância no sexo masculino na faixa etária entre 20 e 40 anos (Soares et al., 2010).

Os principais risco ocupacionais são: trabalhadores na área da limpeza de esgotos, das fazendas, garis e médicos veterinários, devido ao contato direto (Hartskeerl, 2006). Dessa forma, resultando em grande impacto socioeconômico pelo afastamento dos trabalhadores e alto custo na hospitalização dessas pessoas (Kobayashi, 2005). Com base nessas informações, o objetivo neste trabalho foi investigar a prevalência de casos de leptospirose no estado do Pará no período de 2007 a 2019.

\section{Metodologia}

Realizou-se um estudo descritivo e de caráter e quali-quantitativo (Pereira et al., 2018), por meio de uma retrospectiva epidemiológica da prevalência da Leptospirose no estado do Paraense, na qual a população estimada para o estado corresponde a 8.134.426 habitantes (IBGE, 2021).

Os dados relacionados aos casos de leptospirose foram obtidos por meio do portal do Sistema de Informação de Agravos de Notificação online (SINAN Online), no período de 2007 a 2019, no Estado do Pará.

Realizou-se a análise dos casos por meio do número de casos notificados, sendo considerado as variáveis: sexo (masculino e feminino), faixa etária ( $<1$ ano, 1-4 anos, 5-9 anos, 10-14 anos, 15-19 anos, 20-39 anos, 40-59 anos, 60-64 anos, 65-69 anos, 70-79 anos, >80 anos); escolaridade (AFB: Analfabeto, EFI: Ensino Fundamental Incompleto, EFC: Ensino Fundamental Completo, EMI: Ensino Médio Incompleto, EMC: Ensino Médio Completo, ESI: Ensino Superior Incompleto e ESC: Ensino Superior Completo) e os critérios de confirmação.

Os dados foram tabulados e organizados em planilhas do Microsoft excel®, sendo expostos os dados percentuais referentes a cada análise.

\section{Resultados}

Na Tabela 1 é possível observar o número de casos notificados por ano, contata-se que durante o período de 2007 a 2019 foram registrados 1.642 casos, sendo que o maior índice de registros foi evidenciado nos anos de 2014 (149), seguido por 2008 (144), 2018 (144) e 2019 (144). 
Research, Society and Development, v. 10, n. 12, e599101220902, 2021

(CC BY 4.0) | ISSN 2525-3409 | DOI: http://dx.doi.org/10.33448/rsd-v10i12.20902

Tabela 1. Ano de confirmação dos casos de leptospirose no período de 2007 a 2019.

\begin{tabular}{cc}
\hline Ano Notificação & Casos Confirmados \\
\hline 2007 & 106 \\
2008 & 144 \\
2009 & 107 \\
2010 & 95 \\
2012 & 132 \\
2013 & 103 \\
2014 & 126 \\
2015 & 149 \\
2016 & 133 \\
2017 & 125 \\
2018 & 134 \\
2019 & 144 \\
Total & 144 \\
\hline
\end{tabular}

Fonte: Autores.

Em relação ao critério utilizado para a confirmação dos casos de leptospirose, evidenciou-se que a maior parte dos casos foram confirmados por meio dos aspectos clínicos-laboratoriais, com 1.196 casos, seguido pelo critério clinicoepidemiológico.

Tabela 2. Critério de confirmação para os casos de leptospirose de 2007 a 2019.

\begin{tabular}{ccccc}
\hline Ano Notificação & Ign/Branco & $\begin{array}{c}\text { Clínico- } \\
\text { Laboratorial }\end{array}$ & $\begin{array}{c}\text { Clínico- } \\
\text { epidemiológico }\end{array}$ & Total \\
\hline 2007 & 3 & 75 & 28 & 106 \\
2008 & 1 & 105 & 38 & 144 \\
2009 & 1 & 88 & 18 & 95 \\
2010 & - & 72 & 23 & 132 \\
2011 & - & 123 & 9 & 103 \\
2012 & 1 & 84 & 18 & 126 \\
2013 & 2 & 104 & 20 & 149 \\
2014 & 1 & 133 & 15 & 133 \\
2015 & 4 & 83 & 46 & 125 \\
2016 & 4 & 72 & 49 & 134 \\
2017 & 3 & 88 & 43 & 144 \\
2018 & 2 & 89 & 53 & 144 \\
2019 & - & 80 & 64 & 1642 \\
Total & 22 & 1196 & 424 & 95 \\
\hline
\end{tabular}

Fonte: Autores. 
O sexo masculino foi o mais afetado pelos casos de leptospirose no período de 2007 a 2019, alcançando 1.264 casos ao comparar com o feminino, com apenas 378 casos, conforme observado na Tabela 3 . O maior índice de casos para o sexo masculino e feminino foi identificado nos anos de 2019 (119) e 2016 (42).

Tabela 3. Gênero para os casos de leptospirose de 2007 a 2019.

\begin{tabular}{cccc}
\hline Ano Notificação & Masculino & Feminino & Total \\
\hline 2007 & 79 & 27 & 106 \\
2008 & 125 & 19 & 144 \\
2009 & 87 & 20 & 107 \\
2010 & 73 & 22 & 95 \\
2011 & 101 & 31 & 132 \\
2012 & 64 & 39 & 103 \\
2013 & 97 & 29 & 126 \\
2014 & 111 & 38 & 149 \\
2015 & 110 & 23 & 133 \\
2016 & 83 & 42 & 125 \\
2017 & 105 & 29 & 134 \\
2018 & 110 & 34 & 144 \\
2019 & 119 & 25 & 144 \\
Total & 1264 & 378 & 1642 \\
\hline
\end{tabular}

Fonte: Autores.

Avaliando os dados relacionados a escolaridade, observamos que as pessoas consideradas analfabetas (598 casos) apresentam maior número de casos de leptospirose e com menor ocorrência da enfermidade estão as pessoas com ensino superior completo com 16 casos (Tabela 4). 
Tabela 4. Escolaridade para os casos de leptospirose de 2007 a 2019.

\begin{tabular}{cccccccccc}
\hline Ano & AFB & 1 a 4 & $>4$ & 5 a 8 & EFC & EMI & EMC & ESI & ESC \\
& & EFI & EFI & EFI & & & & & \\
\hline 2007 & 53 & - & 8 & 15 & 10 & 7 & 6 & 7 & - \\
2008 & 41 & 1 & 11 & 22 & 29 & 15 & 11 & 6 & 4 \\
2009 & 53 & 1 & 13 & 7 & 10 & 9 & 5 & 6 & - \\
2010 & 52 & 2 & 7 & 5 & 14 & 5 & 2 & 5 & - \\
2011 & 32 & 1 & 26 & 9 & 18 & 21 & 10 & 12 & 1 \\
2012 & 33 & 1 & 14 & 5 & 13 & 9 & 10 & 11 & 1 \\
2013 & 36 & 2 & 13 & 4 & 31 & 9 & 14 & 13 & - \\
2014 & 64 & 1 & 14 & 7 & 21 & 4 & 12 & 19 & 1 \\
2015 & 56 & 4 & 10 & 5 & 18 & 10 & 15 & 12 & 1 \\
2016 & 42 & - & 15 & 6 & 11 & 9 & 10 & 26 & 1 \\
2017 & 48 & 3 & 14 & 2 & 21 & 9 & 6 & 21 & 2 \\
2018 & 44 & 2 & 14 & 4 & 19 & 7 & 14 & 25 & 4 \\
2019 & 44 & 2 & 21 & 6 & 26 & 8 & 8 & 21 & 1 \\
Total & 598 & 20 & 180 & 97 & 241 & 122 & 123 & 184 & 16 \\
\hline
\end{tabular}

*AFB: Analfabeto, EFI: Ensino Fundamental Incompleto, EFC: Ensino Fundamental Completo, EMI: Ensino Médio Incompleto, EMC: Ensino Médio Completo, ESI: Ensino Superior Incompleto e ESC: Ensino Superior Completo. Fonte: Autores.

Para a classificação da faixa etária observou-se que a média de idade de maior incidência se situou 20 a 39 anos, com 663 casos. De outro modo, o intervalo de idade de menor incidência foi para pacientes com idade igual ou superior a 80 anos (4), seguido pelos grupos etários menos que um ano (10 casos) e entre 1-4 anos (13 casos) (Tabela 5).

\section{Discussão}

Neste estudo, a Leptospirose atinge predominantemente o sexo masculino, corroborando com outros autores, que destacam que as características próprias do sexo masculino favorecem o maior contato com o patógeno, ou seja, apresentam uma maior vulnerabilidade (Vasconcelos et al., 2012). Em estudo realizado por Peric et al. (2005), sobre a ocorrência de casos de Leptospirose, no período de 1969 - 2003, identificou-se que 85\% dos casos registrados eram do sexo masculino (Soares et al., 2010; Gonçalves et al., 2016; Magalhães \& Acosta, 2019; Rodrigues et al., 2019).

O registro de casos confirmados, é a principal fonte de explicação sobre a diferença no número de casos entre os sexos encontrada neste trabalho, sendo um reflexo do sistema de vigilância que acaba obtendo os casos graves da doença (Souza et al., 2007), outra possível explicação é que os homens procuram assistência médica quando a doença se apresenta com sintoma mais graves, enquanto as mulheres procuram no estágio inicial (Borghi \& Queiroz, 2017).

Este estudo evidenciou-se que a faixa etária de 20 - 39 anos foi a mais acometida pela leptospirose. De acordo com Souza et al. (2007), esse grupo etário e composto de indivíduos em plena atividade social, dessa forma, pode relacionar com as condições de higiene não adequadas no local de trabalho (Jasen et al., 2007). Resultados diferentes foram encontrados por Everard et al. (1984), que relataram a ocorrência da leptospirose em pessoas acima de 50 anos, corroborando também com Gonçalves et al. (2016), que relataram em seu estudo a maior ocorrência da doença na faixa etária de 15 a 50 anos e, por fim Kawaguchi et al. (2008) que observaram maior incidência em pessoas acima de 15 anos. 
As crianças e os idosos foram os grupos etários com menor ocorrência de leptospirose, sendo as crianças com idade abaixo de 10 anos e idosos acima de 80 anos. Semelhante aos resultados encontrados em países como Trinidad (Everard et al., 1984), Barbados (Everard et al., 1989) e Nova Caledônia (Goarant et al., 2009).

O menor número de casos em idosos da faixa etária de 80 anos, pode ser relacionado à imunidade desenvolvida em decorrência das exposições durante a vida aos ambientes endêmicos (Hartskeerl, 2005).

Neste estudo, foi evidente que o critério clinico-laboratorial foi o mais utilizado para a confirmação dos casos de leptospirose. Ressalta-se que as técnicas laboratoriais são as mais utilizadas e recomendadas para o diagnóstico da doença (Pospissil \& Freitas, 2007), sendo que no estudo de Magalhães e Acosta (2019) foram investigados 228 casos, deste, 86,4\% foram confirmados por meio do critério clínico-laboratorial. Por outro lado, em casos que não puderam ser diagnosticados por métodos laboratoriais, utiliza-se o critério clínico-epidemiológico (Juglar, 2005).

\section{Conclusão}

Com base nessas informações, foi possível identificar que o ano de maior ocorrência de casos foi 2014 com elevado número de casos confirmados. O grupo etário mais evidente foi pessoas do sexo masculino, com faixa etária de 20 a 39 anos, sendo o critério de confirmação clinico-laboratorial o mais adotado.

\section{Referências}

Borghi, A. F. R., \& Queiroz, S. J. (2017). Distribuição da leptospirose humana no Brasil. Estudos Vida e Saúde, 44(1): 115-123.

Everard, C. O. R., Edwards, C. N., Webb, G. B., White, H. S., \& Nicholson, G. D. (1984). The prevalence of severe leptospirosis among humans on Barbados. Transactions of the Royal Society of Tropical Medicine, 78(5): 596 - 603. 10.1016/0035-9203(84)90216-5

Everard, C. O. R., Hayes, R. J., \& Edwards, C. N. (1989). Leptospiral infection in school-children from Trinidad and Barbados. Epidemiology and Infection, 103(1): 143-56. 10.1017/S0950268800030442

Goarant, C., Laumond-Barny, S., Perez, J., Vernel-Pauillac, F., Chanteau, S., \& Guigon, A. (2009). Outbreak of leptospirosis in New Caledonia: diagnosis issues and burden of disease. Tropical Medicine \& International Health, 14(8): 1-4. 10.1111/j.1365-3156.2009.02310.x

Gonçalves, N. V., et al. (2016). Distribuição espaço-temporal da leptospirose e fatores de risco em Belém, Pará, Brasil. Ciência \& Saúde Coletiva, 21(12): 3947-3955. Disponível em: <https://doi.org/10.1590/1413-812320152112.07022016>.

Hartskeerl, R. A. (2005). International Leptospirosis society: objectives and archievements. Revista cubana de medicina tropical, 57(1): 7-10.

Hartskeerl, R. A. (2006). Leptospirosis: current status and future trends. Indian Journal of Medical Microbiology, $24: 309$.

Jansen, A., Luge, E., Guerra, B, Wittschen, P., Gruber, A. D, Loddenkemper, T. S., Lierz, M., Ehlert, D., Appel, B., Stark, K., \& Nockler, K. (2007). Leptospirosis in Urban Wild Boards, Berlim, Germany. Infectious diseases, 13(5):739-742.

Jouglar, S. D. D. (2005). Diagnóstico de Leptospirose por PCR e Caracterização de Isolados de Leptospira spp. [Tese] - Universidade Federal de Pelotas. PELOTAS. Rio Grande do Sul, 2005.

Kawaguchi, L, Sengkeopraseuth, B, Tsuyuoka, R, Koizumi, N, Akashi, H, Vongphrachanh, P, et al. (2008). Seroprevalence of leptospirosis and risk factor analysis in flood-prone rural areas in Lao PDR. American Journal of Tropical Medicine and Hygiene, 78(6): 957-961.

Kobayashi, Y. (2005). Human leptospirosis: management and prognosis. Journal of Postgraduate Medicine. 51(3): 201-4.

Lupi, O, Carneiro, C. G, Coelho, I. C. B. (2007). Manifestações mucocutâneas da dengue Anais Brasileiros de Dermatologia, 82(4): 291-305.

Magalhães, V. S, Acosta, L. M. W. (2019). Leptospirose humana em Porto Alegre, Rio Grande do Sul, de 2007 a 2013: caracterização dos casos confirmados e distribuição espacial*. Revista Epidemiologia e Serviços de Saúde, 28(2): 1-12.

Peric, L., Simasek, D., Barbie, J., Peric, N., Prus, V., Sisljagic, V., et al. (2005). Human leptospirosis in eastern Croatia, 1969-2003: Epidemiological, clinical, and serological features, Scandinavian Journal of Infectious Diseases. 37: 738-41.

Pereira A. S. et al. (2018). Metodologia da pesquisa científica. UFSM.

Picardeau, M. (2017). Virulence of the zoonotic agent of leptospirosis: still terra incognita? Nature Reviews Microbiology, 15(5): $297-307$.

Pospissil, L., \& Freitas, T. T. (2007). Leptospirose Equina. [Monografia]. Faculdade de Jaguariuna. 
Research, Society and Development, v. 10, n. 12, e599101220902, 2021

(CC BY 4.0) | ISSN 2525-3409 | DOI: http://dx.doi.org/10.33448/rsd-v10i12.20902

Rodrigues, M. B. P., Freire, H. B. M., Corrêa, P. R. L., Mendonça, M. L., Silva, M. R. I., \& França, E. B. (2005). É possível identificar a dengue em crianças a partir do critério de caso suspeito preconizado pelo Ministério da Saúde? Journal of Pediatrics, 81(3): 209-215.

Rodrigues, A. L. (2019). Perfil epidemiológico de pacientes acometidos por leptospirose em um estado brasileiro na Amazônia Ocidental. Revista Sustinere, $7(1): 32-45$

Soares, T. S. M., Latorre, M. R. D. O., Laporta, G. Z., \& Buzzar, M. R. (2010). Spatial and seasonal analysis on leptospirosis in the municipality of São Paulo, Southeastern Brazil, 1998 to 2006. Revista Saúde Pública, 44(2): 2-9.

Soo, Z. M. P., Khan, N. A., Siddiqui, R. (2020). Leptospirosis: Increasing importance in developing countries. Acta Tropica, 2020; 201: 105-183.

Souza, V. M. M., Brant, J. L., Arsky, M. L. S., \& Araujo, W. N. (2010). Avaliação do sistema de vigilância epidemiológica da leptospirose Brasil, 2007. Cadernos Saúde Coletiva, 18(1): 95-105.

Vasconcelos, C. H., Fonseca, F. R., Lise, M. L. Z., \& Arsky, M. L. N. S. (2012). Fatores ambientais e socioeconômicos relacionados à distribuição de casos de leptospirose no Estado de Pernambuco, Brasil, 2001-2009. Cadernos Saúde Coletiva, 20(1). 